\title{
Uniportal thoracoscopic surgery: from medical thoracoscopy to non-intubated uniportal video-assisted major pulmonary resections
}

\author{
Diego Gonzalez-Rivas ${ }^{1,2}$ \\ ${ }^{1}$ Minimally Invasive Thoracic Surgery Unit (UCTMI), Coruña, Spain; ${ }^{2}$ Department of Thoracic Surgery, Coruña University Hospital, Coruña, Spain \\ Correspondence to: Diego Gonzalez-Rivas. Department of Thoracic Surgery, Coruña University Hospital, Xubias 84, 15006 Coruña, Spain. Email: \\ diego.gonzalez.rivas@sergas.es.
}

\begin{abstract}
The development of thoracoscopy has more than one hundred years of history since Jacobaeus described the first procedure in 1910. He used the thoracoscope to lyse adhesions in tuberculosis patients. This technique was adopted throughout Europe in the early decades of the $20^{\text {th }}$ century for minor and diagnostic procedures. It is only in the last two decades that interest in minimally invasive thoracic surgery was reintroduced by two key technological improvements: the development of better thoracoscopic cameras and the availability of endoscopic linear mechanical staplers. From these advances the first video-assisted thoracic surgery (VATS) major pulmonary resection was performed in 1992. In the following years, the progress of VATS was slow until studies showing clear benefits of VATS over open surgery started to be published. From that point on, the technique spread throughout the world and variations of the technique started to emerge. The information available on internet, live surgery events and experimental courses has contributed to the rapid learning of minimally invasive surgery during the last decade. While initially slow to catch on, the traditional multi-port approach has evolved into a uniportal approach that mimics open surgical vantage points while utilizing a non-rib-spreading single small incision. The early period of uniportal VATS development was focused on minor procedures until 2010 with the adoption of the technique for major pulmonary resections. Currently, experts in the technique are able to use uniportal VATS to encompass the most complex procedures such as bronchial sleeve, vascular reconstructions or carinal resections. In contrast, non-intubated and awake thoracic surgery techniques, described since the early history of thoracic surgery, peaked in the decades before the invention of the double lumen endotracheal tube and have failed to gain widespread acceptance following their re-emergence over a decade ago thanks to the improvements in VATS techniques.
\end{abstract}

Keywords: Thoracoscopy; advances in video-assisted thoracic surgery (VATS); minimally invasive surgery; uniportal VATS; surgical improvements

Submitted Feb 23, 2016. Accepted for publication Mar 02, 2016.

doi: $10.21037 /$ acs.2016.03.07

View this article at: http://dx.doi.org/10.21037/acs.2016.03.07

\section{Background}

The uniportal approach to diagnosing and treating thoracic disease has existed for much longer than most thoracic surgeons care to acknowledge. Under the umbrella of pleuroscopy and thoracoscopy, physicians have been using scopes and a single port incision to inspect, biopsy and perform thoracic procedures since Hans Christian Jacobaeus first described this procedure (1) in 1910 to treat pleural adhesions $(2,3)$, calling it thoracoscopy. While there are anecdotal reports of thoracoscopy use as early as 1866 , Jacobaeus is widely acknowledged as the inventor of this technique. Notably, the pioneers of this approach usually performed thoracoscopy or pleuroscopy in spontaneously breathing patients using a local anesthetic (4).

Around the world, under the terminology of 'pleuroscopy', many thoracoscopic procedures often fall under the domain of pulmonologists, away from the supervision of thoracic surgeons $(5,6)$. This does not lessen the importance of this technique to the development of current advancements in 
the uniportal approach (7).

\section{The development of medical thoracoscopy}

Jacobaeus continued his work using thoracoscopy, i.e., the "Jacobaeus Operation", to treat adhesions, infections and effusions in addition to diagnosing tuberculosis, tumors and other pathology well into the 1920s. To perform thoracoscopy, he developed both a dedicated cystoscope and a dual port approach for visualization (8). His diagnostic thoracoscopies aided in our understanding of the normal and diseased appearances of the lung and pleural cavity (4). His counterparts in the 1920s, mainly Cutler, Davidson, Friedel and Drash, focused on uniportal techniques. Drash and others published data based on large patient studies in the 1930's (3). Others, such as Sattler, Kux and Roche, expanded the use of thoracoscopy to include treatment of spontaneous pneumothorax, sympathectomy and talc pleurodesis (5).

Even after the advent of antibiotics resulted in therapeutic thoracoscopy largely falling from favor, advances in anesthesia and intra-operative oxygenation made thoracoscopic biopsy an invaluable tool before falling into disuse (4).

The emergence of modern thoracoscopy was just beginning to revive these forgotten techniques. Upon rediscovery, many thoracic surgeons were readily embracing thoracoscopy, especially with the advent of modern technology, such as video displays, which allowed the surgeon to see through the thoracoscope as never before.

\section{Evolution to video-assisted thoracoscopic surgery}

Interest in minimally invasive thoracic surgery was rekindled in the last few decades by two key technological breakthroughs: (I) the development of video-assisted cameras offering a panoramic view of the hemithorax instead of the previous tunnel-like view with direct vision and (II) the availability of new endoscopic instruments like the linear mechanical stapler. From these advances, video-assisted thoracic surgery (VATS) was born (9). Since the first VATS major pulmonary resection was reported in in 1992 (10), thoracoscopy has regained its relevance to thoracic surgery. While the adoption of VATS was initially slow, it has now become the standard of care for many procedures (11).

With the re-emergence of thoracoscopy, surgeons utilized a multi-port approach, like Jacobaeus, with one port used for visualization and additional ports for instrumentation. As this technique was further developed for more complex operations, the initial port configurations were further refined for better ergonomics, visualization and access to anatomical structures (7). Later, as VATS became more popular within thoracic surgery, further development brought two-port configurations, robotic assisted surgery and needlescopic approaches. VATS was also introduced into use for increasingly complex operations including mediastinal masses, large resections and cases with chest wall involvement (12). The old rules regarding absolute contraindications for VATS and the standard indications for open thoracotomy were broken with excellent results. Eventually, as the nature of the evidence changed from case reports, to retrospective studies, to randomized trials, and finally to large scale meta-analyses, VATS began to replace the open thoracotomy as the standard approach in international guidelines and day-to-day practice (13).

\section{The rise of uniportal VATS (Video 1)}

Uniportal VATS started from humble beginnings, with an initial report of uniportal VATS thoracic sympathectomy in 2000 (14). By pushing the boundaries of what might be possible with a uniportal approach, this initial publication was the stimulus for the development of more complex uniportal procedures to treat an ever-increasing range of thoracic conditions.

The results of the first prospective trial reporting the method to perform several thoracic procedures through a uniportal VATS technique was published in 2000, 2001 and 2003 (15-17).

Rocco was one of the first thoracic surgeons to break through the remaining barrier to bring thoracic surgery almost full circle, back to the uniportal approaches of the 1920s to the time of Drash, Cutler and Freidel. Advances in visualization with the use of large sized monitors and high performance fiber-optics have now made earlier challenges with this technique easily surmountable. Rocco popularized the approach, utilizing a uniportal approach for minor procedures such as pneumothorax and wedge resections and reported it in several publications in the early 2000s (18-20). The work of Rocco was further enhanced by our team with the development of the uniportal approach for lobectomy (21). Initially the technique was described only for lower lobes but it was rapidly improved and developed for upper lobes, segmentectomy and pneumonectomy (22-25). In quick succession, more difficult cases were reported including bronchial sleeve resection, pulmonary artery resection and reconstruction, and then, in 2014, a 
double sleeve resection (26-30). As the uniportal approach was successfully applied to more and more complex cases, the contraindications for the uniportal approach changed. Tumors with chest wall involvement slipped out of absolute contraindication to become more of a general surgical consideration after multiple chest wall resection cases were successfully performed (31). The same expansion occurred with the patient selection criteria as it quickly become apparent that general anesthesia was one of the most limiting factors for surgery. This led to the development of a non-intubated protocol that has allowed the uniportal approach to be used for patients who were otherwise ineligible or in a high risk category for thoracic surgery (32-34).

\section{The speed of the adoption of uniportal VATS}

As previously mentioned, the early period of uniportal VATS development was focused on minor procedures (from 2000 to 2010). The second phase of uniportal VATS began in 2010 with the development of the technique for major pulmonary resections $(35,36)$. In only a period of five years, experts have been able to apply the uniportal VATS technique to encompass more complex procedures such as bronchial sleeve, vascular reconstruction and carinal resection. The information available on the Internet, at live surgery events and experimental courses all contributed to the rapid development of uniportal minimally invasive surgery during the last decade (37).

The sheer volume of publications and the variety of increasingly complex uniportal procedures performed by the pioneers has brought this technique to the forefront of modern thoracic surgery. This, combined with a very active lecture circuit and numerous YouTube surgical publications, hands-on wet lab courses and documentary films have advanced the popularity and adoption of this uniportal approach at a speed unheard of in modern medicine. Part of the rapidity of the progression of minimally invasive surgery in an age of internet and global connectivity can be attributed to enhanced collaborative efforts within the international thoracic community. In today's thoracic surgery, developments and accomplishments can be shared in an instant, with on-line video demonstrations. No longer is the majority of information shared via paper publications or at annual conferences. On-line journal editions now allow interested readers to comment and question the authors weeks before the paper journal is mailed out to subscribers.

\section{Philosophical shift}

The development and adoption of uniportal VATS on a widespread scale globally requires a shift in current philosophies and treatment algorithms for common thoracic conditions. Empyema, for example, has traditionally been treated according to guidelines that were developed when open thoracotomy was the standard of care. The morbidity and mortality associated with this approach relegated surgical intervention to the last line of treatment after the failure of less effective and less invasive treatments (38). In an era when open thoracotomy has become a rare exception, rather than the rule, the application of "surgery as a last resort" leads to unnecessary delays that increase the morbidity, mortality and suffering of our patients (39). In the treatment of lung cancer, this has been addressed to some extent within thoracic surgery and pulmonology with the most recent clinical guidelines (40). However, the terminology and use of non-thoracic surgeons as the decision-maker for determining the patient's eligibility for surgery may require re-exploration now that uniportal and related non-intubated anesthetic approaches have been used safely and effectively on even the frailest patients $(32,41)$.

Non-intubated and awake thoracic surgery techniques, described since the early history of thoracic surgery, peaked in the decades before the invention of the dual lumen endotracheal tube and have failed to gain widespread acceptance following their re-emergence over a decade ago (42).

A discussion of non-intubated thoracic surgery and minimally invasive VATS surgery is a discussion involving two distinct phenomena: anesthetic technique and surgical technique. Despite this, both surgical and anesthetic advances share commonalities, particularly from a historical perspective. Both suffer from the phenomenon of forgotten knowledge, having been initially discovered and used successfully before being forgotten and subsequently rediscovered, advanced and readopted several decades later. Both techniques have also suffered from significant skepticism upon their re-emergence, which has hindered rapid widespread adoption.

Recently the aim to avoid intercostal nerve damage incurred by the transthoracic incision during VATS has led to the development of a novel procedure entitled the uniportal VATS subxiphoid approach. To attempt the subxiphoid approach, it is mandatory to have both a previous experience in uniportal VATS lobectomy and a skilled assistant. The subxiphoid approach technique has several limitations such as the difficulty of controlling 
major bleeding and performing a complete oncologic lymph node dissection. The access for the view of the posterior mediastinum is challenging, which makes the complete dissection of the subcarinal space very difficult to accomplish. Despite these disadvantages, this novel approach has the potential for widespread use in the future after the development of new technology such as wireless cameras, better instruments or single port robotic technology adapted to the subxiphoid approach.

While common philosophical approaches to thoracic disease have changed within the thoracic surgical specialty, the paradigm shift has not yet made its way to general medicine and to the general practitioners who still serve as the gatekeepers to our patient populations. Too often, referrals for curative surgeries are delayed or omitted while general practitioners mull over whether frail or debilitated patients are eligible surgical candidates. When this surgical candidacy is weighed against open thoracotomy or sternotomy, patients with significant co-morbidities such as oxygen-dependent chronic obstructive pulmonary disease or coronary artery disease, who are likely to have been successfully treated with minimally invasive techniques, are instead shunted to less effective therapies such as chemotherapy, radiation or even palliative therapies. They are thus are more likely to suffer adverse outcomes.

Therefore, it is incumbent among the practitioners within the thoracic surgical specialty to make special efforts to educate practitioners in other specialty areas, through the publication of clear treatment algorithms and evidencebased practice guidelines.

\section{Basic principles of the uniportal technique}

Uniportal VATS represents a drastic change in the approach to lung resection compared to conventional multiport VATS as the placement of the surgical instruments and the camera are both done through the same incision. Recent industry improvements such as the specifically designed instruments with double articulation, ultra high definition video-camera systems, new energy devices and narrower and more angulated staplers, have made uniportal VATS for major lung resections easier to learn and safer.

The spread of uniportal VATS oncologic surgery has been possible because it has been shown that oncologic principles can be achieved by this approach without compromising the safety of the patient $(43,44)$. It is essential that during the dissection of structures, the basic principles are respected and followed when performing individual dissection of veins, arteries and bronchus, as well as completion of an appropriate lymphadenectomy $(45,46)$.

During the uniportal VATS procedure, both the surgeon and the assistant are usually positioned in front of the patient in order to have the same thoracoscopic vision throughout the procedure. Even though the field of vision can only be obtained through the anterior access site, movement of a 30-degree thoracoscope along the incision allows for different angles of vision. The role of the camera assistant is important during any VATS procedure but it is specially demanding during uniportal VATS because this technique lacks the dedication of one port for the camera that is usual for multiport VATS techniques. The advantage of using the thoracoscope in the same port and in coordination with the instruments is that vision is directed to the target tissue. By doing this, we are lining up the instruments to address the target lesion from a direct, sagittal perspective (43). Optimal exposure of the lung is vital in order to facilitate the dissection of structures and to avoid any instrument interference.

Uniportal VATS is performed with the patient positioned in a lateral decubitus position that is the same as for a conventional multiport VATS procedure. The access incision, about $3-4 \mathrm{~cm}$ long, is preferably made in the anterior fifth intercostal space. This incision location provides better angles for hilar dissection and insertion of staplers. It is helpful to rotate the surgical table away from the surgeon during the hilar dissection and division of structures and towards the surgeon for the subcarinal lymph node dissection. We always recommend inserting the staplers, with angulation, through the anterior part of the incision. The use of curved-tip stapler technology allows for improved placement around the superior pulmonary vein and bronchus through a single incision, which is very helpful as these are the most difficult structures to divide through a single port. It is important to dissect the vessel as distal as possible in order to achieve better angles for the stapler insertion. When the angle is difficult for stapler insertion, we either use polymer vascular clips or ligate the vessels by using sutures.

It is crucial that the thoracoscope remains positioned in the posterior part of the incision at all times and the instruments in the anterior part as this positioning provides the best view and functionality. In the case of upper lobectomies, the pulmonary artery is normally divided first, followed by the vein. When the lobectomy is completed, the lobe is removed in a protective bag and a systematic lymph node dissection is accomplished. At the end of the surgery, the intercostal spaces are infiltrated with bupivacaine under 
thoracoscopic view and a single-chest tube is placed in the posterior part of the incision.

\section{The role of the uniportal technique for advanced cases}

With experience, skilled uniportal VATS surgeons can perform the most complex cases in the same manner as surgeons using a double or triple port approach $(47,48)$. Previous experience in VATS is necessary to perform these advanced cases with success. The experience acquired with minimally invasive techniques such as uniportal VATS and improvements in surgical instruments, high definition cameras and recent three-dimensional systems have greatly facilitated the adoption of uniportal VATS techniques for complex cases and sleeve reconstructions. We believe it is important to minimize the surgical aggressiveness, especially in advanced stage lung cancer patients where the immune system is weakened by the disease or by induction treatments. The thoracoscopic surgical approach represents the least invasive technique to operate on lung cancer and the uniportal technique is the final stage in the evolution of these minimally invasive surgical techniques. The geometrical concept of the uniportal approach, i.e., the ergonomy obtained with the direct view and bimanual instrumentation could explain the excellent results that we have obtained with this technique for sleeve procedures $(49,50)$. Moreover, this geometric ergonomy also contributes to the easy learning of the technique by numerous surgeons, many of them directly transitioning from open surgery to the uniportal VATS technique.

However, despite the multiple advantages of conventional multiport or uniportal VATS compared to thoracotomy such as decreased postoperative pain, decreased hospitalization, diminished inflammatory response or faster access to chemotherapy, the uniportal VATS approach for advanced stages of lung cancer is still infrequently undertaken (41). The main reasons for this low adoption of the technique are concerns about an intraoperative thoracoscopic major bleeding event or the technical challenges of performing a radical oncologic resection by uniportal VATS in advanced cases (51).

The majority of the complications that occur during VATS can be minimized with correct preoperative planning of the case as well as a careful pulmonary dissection. Coordination of all the surgical team is essential when confronting a challenging case or an emergency such as major bleeding (52). This is particularly important during the VATS learning curve, where the occurrence of intraoperative complications, particularly significant bleeding, usually ends in a conversion to open surgery. The conversion to open surgery should never be considered as a failure of VATS but rather as a form of guaranteeing the oncologic principles and the safety of the patient, especially for inexperienced thoracoscopic surgeons. It is essential to know at which moment the surgery should be converted and this will greatly depend on the experience of each surgeon (53). The advantage of the uniportal approach is the speed at which it can be converted to an open thoracotomy. Since the incision is in the anterior fifth intercostal space, it just needs to be enlarged posteriorly and a rib spreader introduced. When converting to a thoracotomy for bleeding, it is important to always maintain compression and the thoracoscopic view while performing the thoracotomy in order to avoid situations of massive bleeding.

Further studies will be necessary to evaluate the long term results of a large series of complex resections performed by uniportal VATS $(41,54)$.

\section{Conclusions}

The rapid rise of uniportal VATS is over a century in the making. Dr. Jacobaeus and his counterparts provided the foundations from what later become VATS and uniportal surgery. Without the advent of both video fiber optics and the Internet, modern uniportal surgery might still be taking baby steps on to the surgical stage. Now that uniportal surgery has progressed beyond its infancy, it is time to propose changes in existing treatment algorithms to reflect its existence. To do so, thoracic surgery needs to generate additional publications beyond the level of case reports and retrospective review.

\section{Acknowledgements}

The author wishe to thanks the medical writer Kristin Eckland for the contribution to the historical review of thoracoscopy included in this keynote lecture.

\section{Footnote}

Conflicts of Interest: The author has no conflicts of interest to declare.

\section{References}

1. Jacobaeus H. Über die Möglichkeit die Zystoskopie bei 
Untersuchung seröser Höhlungen anzuwenden. Munch Med Wochenschr 1910;57:2.

2. Braimbridge MV. The history of thoracoscopic surgery. Ann Thorac Surg 1993;56:610-4.

3. Litynski GS. Laparoscopy--the early attempts: spotlighting Georg Kelling and Hans Christian Jacobaeus. JSLS 1997;1:83-5.

4. Loddenkemper R, Mathur PN, Lee P, et al. History and clinical use of thoracoscopy/pleuroscopy in respiratory medicine. Breathe 2011;8:91-4.

5. Prabhu VG, Narasimhan R. The role of pleuroscopy in undiagnosed exudative pleural effusion. Lung India 2012;29:128-30.

6. Thomas PA Jr. A thoracoscopic peek: what did Jacobaeus see? Ann Thorac Surg 1994;57:770-1.

7. Sihoe $\mathrm{AD}$. The evolution of minimally invasive thoracic surgery: implications for the practice of uniportal thoracoscopic surgery. J Thorac Dis 2014;6:S604-17.

8. Jacobaeus HC. The Cauterization of Adhesions in Artificial Pneumothorax Treatment of Pulmonary Tuberculosis under Thoracoscopic Control. Proc R Soc Med 1923;16:45-62.

9. Shah RD, D'Amico TA. Modern impact of video assisted thoracic surgery. J Thorac Dis 2014;6:S631-6.

10. Roviaro G, Rebuffat C, Varoli F, et al. Videoendoscopic pulmonary lobectomy for cancer. Surg Laparosc Endosc 1992;2:244-7.

11. Oparka JD, Yan TD, Walker WS. Twenty years of videoassisted thoracoscopic surgery: The past, present, and future. Thoracic Cancer 2013;4:91-4.

12. Luh SP, Liu HP. Video-assisted thoracic surgery--the past, present status and the future. J Zhejiang Univ Sci B 2006;7:118-28.

13. Ng CS, Lau KK, Gonzalez-Rivas D, et al. Evolution in surgical approach and techniques for lung cancer. Thorax 2013;68:681.

14. Nesher N, Galili R, Sharony R, et al. Videothorascopic sympathectomy (VATS) for palmar hyperhidriosis:summary of a clinical trial and surgical results. Harefuah 2000;138:913-6, 1008.

15. Migliore M, Giuliano R, Deodato G. Video assisted thoracic surgery through a single port. Thoracic Surgery and Interdisciplinary Symposium on the threshold of the Third Millennium. An International Continuing Medical Education Programme. Naples, Italy. 2000:2930. Available online: http://xoomer.virgilio.it/naples2000/ index1.html

16. Migliore M, Deodato G. A single-trocar technique for minimally-invasive surgery of the chest. Surg Endosc 2001;15:899-901.

17. Migliore M, Giuliano R, Aziz T, et al. Four-step local anesthesia and sedation for thoracoscopic diagnosis and management of pleural diseases. Chest 2002;121:2032-5.

18. Rocco G, Martucci N, La Manna C, et al. Ten-year experience on 644 patients undergoing single-port (uniportal) video-assisted thoracoscopic surgery. Ann Thorac Surg 2013;96:434-8.

19. Rocco G, Martin-Ucar A, Passera E. Uniportal VATS wedge pulmonary resections. Ann Thorac Surg 2004;77:726-8.

20. Jutley RS, Khalil MW, Rocco G. Uniportal vs standard threeport VATS technique for spontaneous pneumothorax: comparison of postoperative pain and residual paraesthesia. Eur J Cardiothorac Surg 2005;28:43-6.

21. Gonzalez D, Paradela M, Garcia J, et al. Single-port videoassisted thoracoscopic lobectomy. Interact Cardiovasc Thorac Surg 2011;12:514.

22. Gonzalez D, Delgado M, Paradela M, et al. Uni-incisional video-assisted thoracoscopic left lower lobectomy in a patient with an incomplete fissure. Innovations (Phila) 2011;6:45-7.

23. Gonzalez-Rivas D, de la Torre M, Fernandez R, et al. Single-port video-assisted thoracoscopic left upper lobectomy. Interact Cardiovasc Thorac Surg 2011;13:539-41.

24. Gonzalez-Rivas D, de la Torre M, Fernandez R, et al. Video: Single-incision video-assisted thoracoscopic right pneumonectomy. Surg Endosc 2012;26:2078-9.

25. Gonzalez-Rivas D, Fieira E, Mendez L, et al. Single-port video-assisted thoracoscopic anatomic segmentectomy and right upper lobectomy. Eur J Cardiothorac Surg 2012;42:e169-71.

26. Gonzalez-Rivas D. Single port video-assisted thoracoscopic lobectomy and chest wall resection by posterior incision. Innovations 2012;7:81-4.

27. Gonzalez-Rivas D, Fernandez R, Fieira E, et al. Uniportal video-assisted thoracoscopic bronchial sleeve lobectomy: first report. J Thorac Cardiovasc Surg 2013;145:1676-7.

28. Gonzalez-Rivas D, Delgado M, Fieira E, et al. Single-port video-assisted thoracoscopic lobectomy with pulmonary artery reconstruction. Interact Cardiovasc Thorac Surg 2013;17:889-91.

29. Gonzalez-Rivas D, Fieira E, de la Torre M, et al. Bronchovascular right upper lobe reconstruction by uniportal video-assisted thoracoscopic surgery. J Thorac Dis 2014;6:861-3. 
30. Gonzalez-Rivas D, Delgado M, Fieira E, et al. Double sleeve uniportal video-assisted thoracoscopic lobectomy for non-small cell lung cancer. Ann Cardiothorac Surg 2014;3:E2.

31. Gonzalez-Rivas D, Xie B, Yang Y, et al. Uniportal videoassisted thoracoscopic lobectomy with en bloc chest wall resection. J Vis Surg 2015;1:7.

32. Gonzalez-Rivas D, Bonome C, Fieira E, et al. Nonintubated video-assisted thoracoscopic lung resections: the future of thoracic surgery? Eur J Cardiothorac Surg 2016;49:721-31.

33. Gonzalez-Rivas D, Fernandez R, de la Torre M, et al. Single-port thoracoscopic lobectomy in a nonintubated patient: the least invasive procedure for major lung resection? Interact Cardiovasc Thorac Surg 2014;19:552-5.

34. Gonzalez-Rivas D, Aymerich H, Bonome C, et al. From Open Operations to Nonintubated Uniportal VideoAssisted Thoracoscopic Lobectomy: Minimizing the Trauma to the Patient. Ann Thorac Surg 2015;100:2003-5.

35. Gonzalez-Rivas D. VATS lobectomy: surgical evolution from conventional VATS to uniportal approach. Scientific World Journal 2012;2012:780842.

36. Gonzalez-Rivas D, Paradela M, Fieira E, et al. Singleincision video-assisted thoracoscopic lobectomy: initial results. J Thorac Cardiovasc Surg 2012;143:745-7.

37. Gonzalez-Rivas D, Yang Y, Ng C. Advances in Uniportal Video-Assisted Thoracoscopic Surgery: Pushing the Envelope. Thorac Surg Clinics 2015. Available online: http://www.thoracic.theclinics.com/article/S15474127(15)00127-9/abstract

38. Di Napoli G, Ronzini M, Paradies G. VATS: first step in the parapneumonic empyema*. G Chir 2014;35:146-8.

39. Scarci M, Zahid I, Billé A, et al. Is video-assisted thoracoscopic surgery the best treatment for paediatric pleural empyema? Interact Cardiovasc Thorac Surg 2011;13:70-6.

40. Detterbeck FC, Mazzone PJ, Naidich DP, et al. Screening for lung cancer: Diagnosis and management of lung cancer, 3rd ed: American College of Chest Physicians evidence-based clinical practice guidelines. Chest 2013;143:e78S-92S.

41. Gonzalez-Rivas D, Fieira E, Delgado M, et al. Is uniportal thoracoscopic surgery a feasible approach for advanced stages of non-small cell lung cancer? J Thorac Dis 2014;6:641-8.

42. Chen KC, Cheng YJ, Hung MH, et al. Nonintubated thoracoscopic surgery using regional anesthesia and vagal block and targeted sedation. J Thorac Dis 2014;6:31-6.

43. Gonzalez-Rivas D, Fernandez R, de la Torre M, et al. Thoracoscopic lobectomy through a single incision. Multimed Man Cardiothorac Surg 2012;2012:mms007.

44. Gonzalez-Rivas D, Paradela M, Fernandez R, et al. Uniportal video-assisted thoracoscopic lobectomy: two years of experience. Ann Thorac Surg 2013;95:426-32.

45. Gonzalez-Rivas D, Fieira E, Delgado M, et al. Uniportal video-assisted thoracoscopic lobectomy. J Thorac Dis 2013;5 Suppl 3:S234-45.

46. Delgado Roel M, Fieira Costa EM, González-Rivas D, et al. Uniportal video-assisted thoracoscopic lymph node dissection. J Thorac Dis 2014;6:S665-8.

47. Gonzalez-Rivas D, Fieira E, Delgado M, et al. Uniportal video-assisted thoracoscopic sleeve lobectomy and other complex resections. J Thorac Dis 2014 Oct;6:S674-81.

48. Gonzalez-Rivas D, Yang Y, Stupnik T, et al. Uniportal video-assisted thoracoscopic bronchovascular, tracheal and carinal sleeve resections †. Eur J Cardiothorac Surg 2016;49 Suppl 1:i6-i16.

49. Bertolaccini L, Rocco G, Viti A, et al. Geometrical characteristics of uniportal VATS. J Thorac Dis 2013;5 Suppl 3:S214-6.

50. Bertolaccini L, Viti A, Terzi A. Ergon-trial: ergonomic evaluation of single-port access versus three-port access video-assisted thoracic surgery. Surg Endosc 2015;29:2934-40.

51. Fernández Prado R, Fieira Costa E, Delgado Roel M, et al. Management of complications by uniportal video-assisted thoracoscopic surgery. J Thorac Dis 2014;6:S669-73.

52. Demmy TL, James TA, Swanson SJ, et al. Troubleshooting video-assisted thoracic surgery lobectomy. Ann Thorac Surg 2005;79:1744-52; discussion 1753.

53. Gonzalez-Rivas D, Stupnik T, Fernandez R, et al. Intraoperative bleeding control by uniportal video-assisted thoracoscopic surgery†. Eur J Cardiothorac Surg 2016;49 Suppl 1:i17-i24.

54. Ng CS, Gonzalez-Rivas D, D'Amico TA, et al. Uniportal VATS-a new era in lung cancer surgery. J Thorac Dis 2015;7:1489-91.

Cite this article as: Gonzalez-Rivas D. Uniportal thoracoscopic surgery: from medical thoracoscopy to non-intubated uniportal video-assisted major pulmonary resections. Ann Cardiothorac Surg 2016;5(2):85-91. doi: 10.21037/acs.2016.03.07 